\title{
The roles of RUNX3 in cervical cancer cells in vitro
}

\author{
ZHEN LI $^{1 *}$, PAN FAN ${ }^{1 *}$, MIN DENG $^{2}$ and CHAO ZENG ${ }^{1}$ \\ ${ }^{1}$ Department of Pathology, Guangdong Medical University, Dongguan, Guangdong 523808; \\ ${ }^{2}$ Cancer Hospital and Cancer Research Institute, Guangzhou Medical University, Guangzhou, Guangdong 510095, P.R. China
}

Received October 1, 2017; Accepted March 21, 2018

DOI: $10.3892 / \mathrm{ol} .2018 .8419$

\begin{abstract}
RUNX3 serves an important role in development of various types of human cancer. The purpose of the present study was to investigate the potential biological function of RUNX3 in cervical cancer cells. In the present study, a RUNX3 overexpressed model was constructed in Hecl cells by PCDNA3.1-RUNX3 transfection. Western blot analysis was used to measure RUNX3 expression in cervical cancer cells. Immunofluorescence analysis was performed to examine subcellular localization of RUNX3 in cervical cancer cells. Effects of RUNX3 expression on proliferation, migration and invasion of cervical cancer cells were detected by colony formation assay, wound healing assay and Transwell assay, respectively. Immunofluorescence confirmed the nuclear location of RUNX3 in cervical cancer cell. Result sindicated that upregulation of RUNX3 expression inhibited proliferation, migration and invasion of cervical cancer cells. However, knockdown of RUNX3 expression promoted the proliferation, migration and invasion of cervical cancer cells. Hence, RUNX3 may serve as a tumor suppressor gene in cervical cancer.
\end{abstract}

\section{Introduction}

Cervical cancer is one of the most common malignancies in women. The incidence is second only to that of breast cancer, which also poses a serious threat to women's health (1). In recent years, one study found that the continuous upgrading of screening methods and the popularity of the human papillomavirus vaccine had caused the incidence of both cervical

Correspondence to: Dr Chao Zeng, Department of Pathology, Guangdong Medical University, 1 Xincheng Road, Songshan Lake, Dongguan, Guangdong 523808, P.R. China

E-mail: zengchaosysu@126.com

Dr Min Deng, Cancer Hospital and Cancer Research Institute, Guangzhou Medical University, 78 Hengzhigang Road, Yuexiu, Guangzhou, Guangdong 510095, P.R. China

E-mail: mindeng2016@163.com

*Contributed equally

Key words: RUNX3, cervical cancer, proliferation, invasion cancer cases and mortality to decline. Due to the uneven distribution of resources, the incidence is still rising in developing countries, and the trend may affect younger patients (2). As molecular biology research becomes more sophisticated, more and more genes have been found to be involved in the development of cervical cancer. Finding new molecular targets for the prevention and treatment of cervical cancer could have far-reaching implications.

The RUNX3 tumor suppressor gene was discovered a few years ago. With RUNX1 and RUNX2, it makes up the transcription factor RUNX family (3). RUNX1 is associated with hematopoietic function (4), and RUNX2 is an important bone formation regulator (5). RUNX3 is located on chromosome 1p36.1 and contains a p1 promoter and $\mathrm{p} 2$ promoter (6). Alkaline phosphatase (ALP), a marker of BMP9-induced late osteogenic differentiation was enhanced by the overexpression of RUNX3, whereas it was inhibited by the knockdown of RUNX3 (7). It was first reported to be a tumor suppressor gene in gastric epithelial cells (8), and it was also reported to absent or mutated in a variety of cancers for hemizygous deletions (9), protein mislocalization, epigenetic alterations (10), and histone modifications (11). Suzuki et al (12), initially described the DNA promoter of RUNX3 hypermethylation as a characteristic of breast cancer. Paradoxically, with the discoveries reported by Nevadunsky et al (13) and Lee et al (14), found that RUNX3 took on a growth-stimulating role, which was highly active in ovarian cancer cells, likewise, it even played an oncogenic role in basal cell carcinoma, head and neck squamous cell carcinoma $(15,16)$. Moreover, reports have found that RUNX3 inactivation can be correlated with advanced tumor stage and negative prognosis (17), and acts as a crucial early step in the development of tumors $(18,19)$. To date, our purpose is to preliminarily explore the expression and the biological behavior of RUNX3 in cervical cancer.

\section{Materials and methods}

Cell culture and reagents. RUNX3 antibody were bought from Santa Cruz Biotechnology (sc-376543; Santa Cruz Biotechnology, Inc., Dallas, TX, USA). Hcel and Hela cells, were obtained from Xiangya Medical College Cancer Institute. The cells were cultured in DMEM high glucose medium containing $10 \%$ fetal bovine serum (FBS) and penicillin/streptomycin $(50 \mathrm{U} / \mathrm{ml})$ at $37^{\circ} \mathrm{C}$ in $5 \% \mathrm{CO}_{2}$. The $\mathrm{HCE}$ cell line is misidentified according to: http://iclac.org/wp-content/uploads/ Cross-Contaminations-v8_0.pdf. The cells of HCE1 in our 
study names Hunan cervical epithelial cell line no. 1 (HCE1) was established by Chinese researchers in 2000 and have no possibility of being contaminated by Hela cells.

Construction of plasmids and transfection. RUNX3 mRNA sequences were picked from Genebank to design primers. Then PCR amplification was performed and the PCR product was subjected to $1 \%$ agarose gel electrophoresis and the RUNX3-band was recovered. The recovered RUNX3 fragment and vector PCDNA3.1 were digested with HindIII and XhoI, and the gel was recovered by electrophoresis, then ligated with T4 DNA ligase overnight at $4^{\circ} \mathrm{C}$. At last, the ligation product was transformed into DH5a. After the single clones were picked, the extracted plasmids were identified and sequenced by double digestion. Then Hcel cells were transfected with PCDNA3.1-RUNX3 by Lipofectamine-2000. The cells with RUNX3 overexpression were named as 'Hce1-RUNX3 cells' in this manuscript.

RNA interference. Homo sapiens RUNX3 mRNA sequence (GenBank accession no. NM_001031680.2) was used to design RUNX3 siRNA (Guangzhou Ruibo Biological Technology Co..Ltd., Guangzhou, China). The following base pairs of siRNA were used for RUNX3: siRNA 646, (sense) 5'-CCATCACTG TGTTCACCAATT-3'and (antisense) 3'-TTGGTGAACACAGTG ATGGTT-5'; siRNA 895, (sense) 5'-CCTCGGAACTGAACC CATTTT-3' and (antisense) 5'-AATGGGTTCAGTTCCGAGGT $\mathrm{T}-3^{\prime} ; 100 \mathrm{nM}$ in final concentration; $24 \mathrm{~h}$ after transfection, cells were harvested and used for experiments. Lipofectamine 2000 was used for siRNA transfections. HeLa cells were transfected with siRNA by Lipofectamine-2000. The cells with RUNX3 siRNA were named as 'HeLa-SiRUNX3 cells' in this manuscript.

Western blot analysis. Whole cell lysates containing $30 \mu \mathrm{g}$ of total cellular proteins were analyzed by western blot. The antibodies used include RUNX3 antibody (1:400) and goat anti-mouse HRP labeled antibody, which were purchased from Santa Cruz Biotechnology, Inc.; GAPDH antibody $(1: 4,000)$ was purchased from Hangzhou Sanjian Company. We analyzed the molecular weight and grayscale values of the target bands with the Gelpro 32 analysis software processing system and calculated the RUNX3/GAPDH ratio.

Immunofluorescence. The cells were fixed in $2.5 \%$ paraformaldehyde for $30 \mathrm{~min}$ at room temperature and then permeated with $0.2 \%$ Triton-X for $15 \mathrm{~min}$. Subsequently, the cells were blocked with $3 \%$ BSA/PBS for $30 \mathrm{~min}$ and incubated overnight at $4^{\circ} \mathrm{C}$ with RUNX3. After washing, the cells were incubated with secondary anti-mouse $\operatorname{IgG}$ at room temperature for $1 \mathrm{~h}$. Nuclei of cells were stained with $1 \mu \mathrm{g} / \mathrm{ml}$ of RUNX3, DAPI, and Merger.

Wound healing experiment. Hce-1 cells were overexpressed with RUNX3 and Hela cells were transfected into 24-well plates with siRUNX3. After the cells were cultured and grown to $100 \%$ confluence, they were scratched with a $10 \mu \mathrm{l}$ pipette tip (time 0), washed with PBS to remove isolated cells, and incubated with complete growth medium. The cells migrated to the injured area and were photographed after $48 \mathrm{~h}$ with an inverted microscope.
Transwell assay. Hce-1 cells were overexpressed with RUNX3 and Hela cells were transfected with SiRUNX3, respectively. The polycarbonate film was coated with $1 \mathrm{mg} / \mathrm{ml}$ matrigel gum (dissolved in serum-free DMEM medium) and incubated at $37^{\circ} \mathrm{C}$ for $1 \mathrm{~h}$. Homogeneous cells $\left(2.0 \times 10^{4}\right)$ suspended in $100 \mu 1$ of complete medium were inoculated in the upper chamber of the transwell unit, $700 \mu \mathrm{l}$ of a high-sugar medium containing $10 \%$ FBS was added to the lower chamber as a chemical attractant. These were allowed to invade for $48 \mathrm{~h}$ at $37^{\circ} \mathrm{C}$ in a $\mathrm{CO}_{2}$ incubator. Then the cells above the membrane were removed and the cells migrating through the membrane to the lower chamber were fixed with $75 \%$ ethanol and stained with $0.5 \%$ crystal violet.

Colony formation experiment. After $24 \mathrm{~h}$ of transfection, the cells were seeded at a density of $300 / \mathrm{ml}$ in 6 -well plates. Colonies were allowed to grow for 2 weeks. The culture medium was discarded and washed twice with PBS. Then the cells were fixed in methanol for 15 min and stained with Giemsa solution for $20 \mathrm{~min}$. Lastly, the clones (more than 10 cells) were counted under a microscope.

Statistical analysis. All statistical analyses were done using SPSS 17.0 for windows. The data are presented as the mean \pm SD. The statistical significance of differences was determined by Student's two-tailed t-test for two groups, and one-way ANOVA followed by Student Newman Keuls post hoc test for multiple groups. P-values of $<0.05$ were considered to indicate a statistically significant difference. All the cell experiments were repeated at least three times.

\section{Results}

Expression of RUNX3 in cervical cancer cell lines. The efficiency of exogenous RUNX3 expression and RUNX3 siRNA in cervical cancer cells was verified by western blot analysis. As shown in picture 1, the protein of forced RUNX3 expression was markedly higher than in controls. However, the vector group (NC) and untreated group (UT) exhibited few differences ( $\mathrm{P}>0.05$; Fig. 1A). The ratios of RUNX3/GAPDH were $13.1 \pm 1.2,1.4 \pm 0.2$, and $0.4 \pm 0.1(\mathrm{P}<0.05$; Fig. 1B). RUNX3 gene was successfully knocked down by si895 in Hela cells (Fig. 1C) and the ratio of RUNX3/GAPDH was $0.3 \pm 0.1$, which was markedly lower than in Hela-UT $(1.4 \pm 0.2)$ group $(\mathrm{P}<0.05$; Fig. 1D).

Subcellular location of RUNX3 in cervical cancer cells. We next examined the subcellular localization of RUNX3 in cervical cells using immunofluorescence. As shown in Fig. 2A and B, RUNX3 clearly demonstrated a strong nuclear localization in Hce1-RUNX3 cells and Hela-UT cells, but in other groups, the expression levels of RUNX3 were visibly decreased.

Roles of RUNX3 in migration and invasion of cervical cancer cells. To establish the biological roles of RUNX3 in cervical cancer cells, wound healing assay was carried out to assess cell migration and Transwell assay was set up to investigate effects of RUNX3 on cell invasion. As shown in Fig. 3, Hce1-RUNX3 cells migrated a shorter distance $(2.2 \pm 0.7)$ than in the Hce1-UT (4.2 \pm 1.4$)$ and Hce1-NC groups (3.7 \pm 0.3$)(\mathrm{P}<0.05$; 

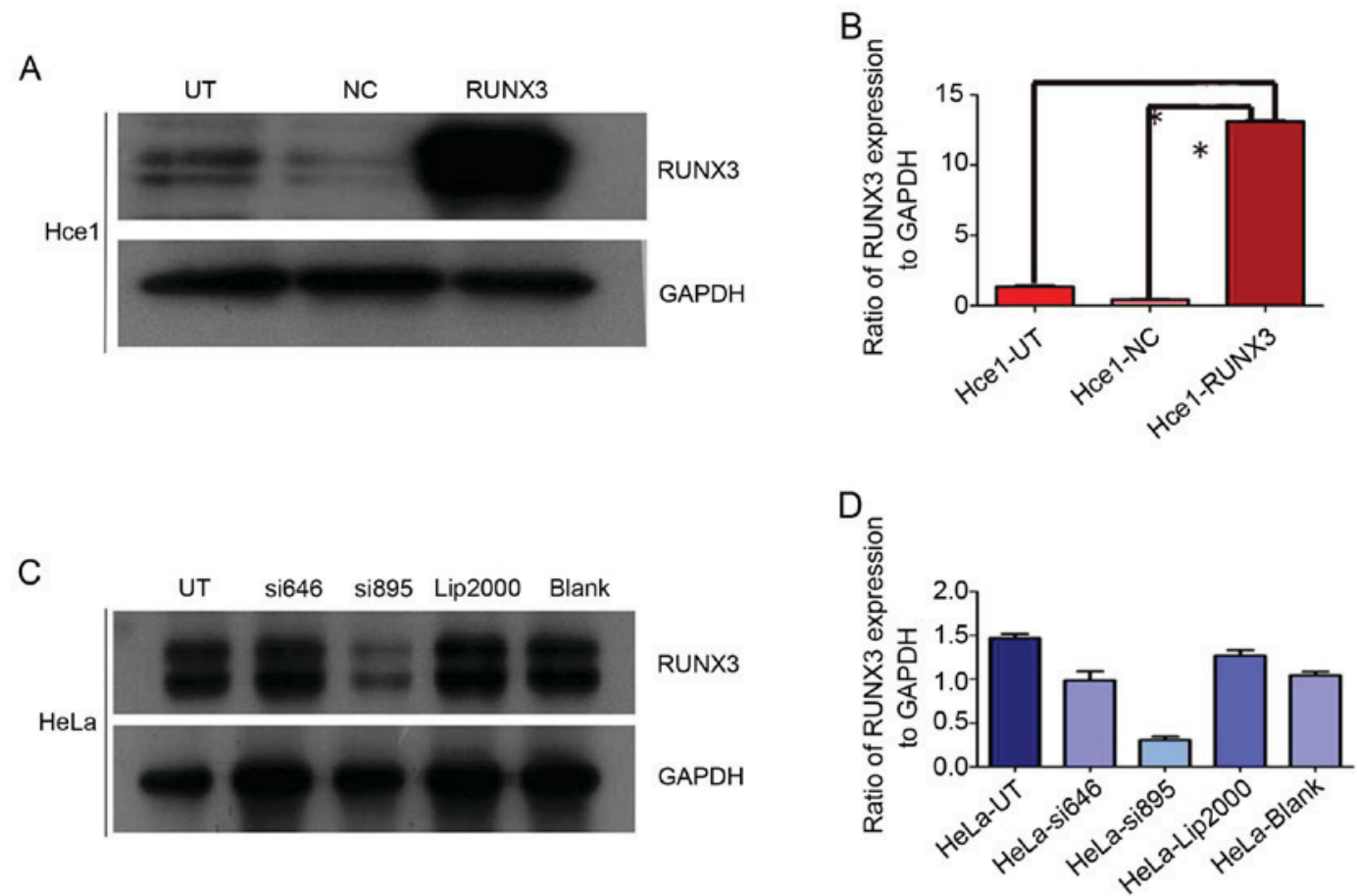

Figure 1. The examination of RUNX3 expression by western blot analysis in cervical cells. (A) Western blot analysis of RUNX3 protein in exogenous infected Hcel cells. (B) The ratio of RUNX3 expression to GAPDH in exogenous transfected Hcel cell. * P $<0.05$. (C) RUNX3 knockdown efficiency of various siRNA were detected by western blot in Hela cell. (D) The ratio of RUNX3 expression to GAPDH in Hela cell. GAPDH was used as loading control.

A
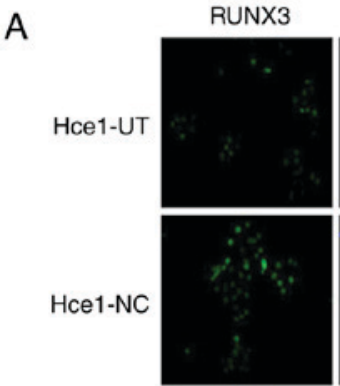

Hce1-RUNX3
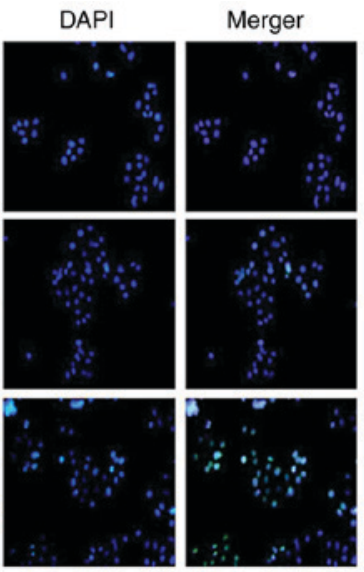

B
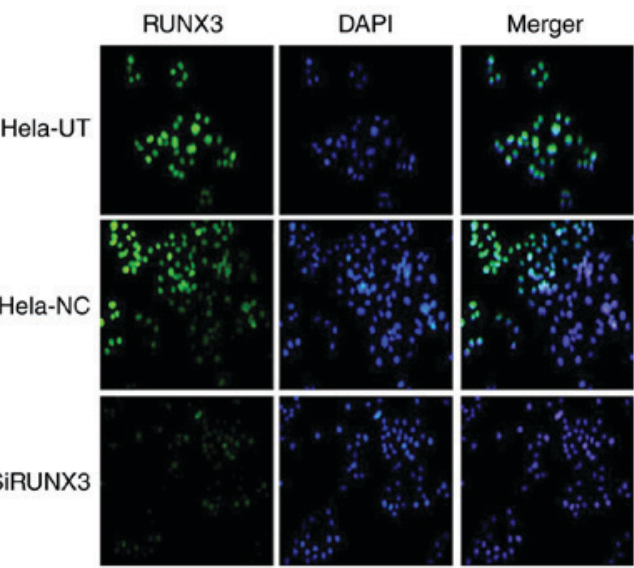

Figure 2. Immunofluorescence analysis of RUNX3 in cervical cancer cells. (A) Immunofluorescence analysis of exogenous infected RUNX3 in Hce1 cell with anti-RUNX3 antibody, nuclei were visualized by staining with DAPI. Hce1-UT and Hce1-NC groups were used as control groups for nuclear RUNX3 staining. (B) Immunofluorescence analysis of RUNX3 expression in HeLa-SiRUNX3, HeLa-UT and HeLa-NC groups. Original magnification, x200. UT, untreated; NC, negative control.

Fig. 3 A and B). Conversely, Hela-SiRUNX3 cells migrated a longer distance $(5.1 \pm 0.6)$ than in the Hela-UT $(2.7 \pm 0.3)$ and Hela-NC groups (2.6 \pm 0.6$)(\mathrm{P}<0.05$; Fig. $3 \mathrm{C}$ and $\mathrm{D})$.

Hcel-RUNX3 cells still showed much lower penetration ability (153 \pm 9$)$ through the matrigel-coated membrane than in the Hcel-UT $(340 \pm 14)$ and Hce1-NC groups $(304 \pm 12)(\mathrm{P}<0.05)$. However, compared with the Hela-UT $(121 \pm 6)$ and Hela-NC groups (130 \pm 8 ), the invasion ability of Hela-SiRUNX3 cell was enhanced (155 \pm 9$)(\mathrm{P}<0.05$; Fig. 4A-D). All of these results collectively suggested that RUNX3 overexpression inhibited cervical cancer cell migration and invasion. However, inhibition of RUNX3 expression promoted migration and invasion of cervical cancer cells.
Role of RUNX3 expression in colony formation. The role of RUNX3 as a tumor suppressor gene was assessed by a test of colony formation assay. Hce1-RUNX3 cells formed $318 \pm 18$ colonies, while the Hce1-UT and Hcel-NC groups formed $433 \pm 16$ and $460 \pm 21$ colonies, respectively $(\mathrm{P}<0.05$; Fig. 5A and B). Conversely, Hela-SiRUNX3 cells had 509 \pm 19 colonies, indicating dramatically greater colony forming ability than the Hela-UT and Hela-NC groups, which formed $402 \pm 14$ and $267 \pm 12$ colonies respectively $(\mathrm{P}<0.05$; Fig. $5 \mathrm{C}$ and D). In this way, these results suggest that stable forced RUNX3 expression can inhibit the ability of cervical cancer cells to form colonies, and silencing RUNX3 expression was found to enhance colony formation by Hela cells. 
A

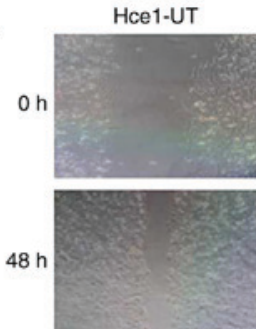

C
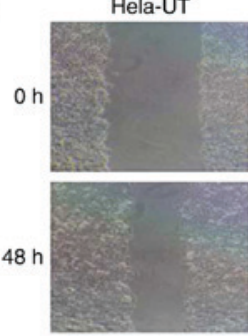

Hce1-NC

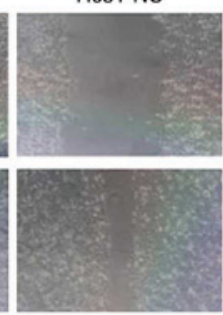

Hela-NC

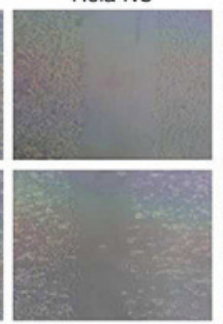

Hce1-RUNX3

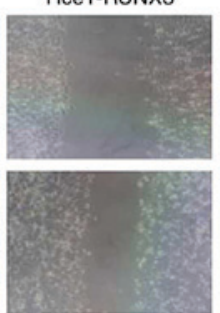

Hela-SiRUNX3

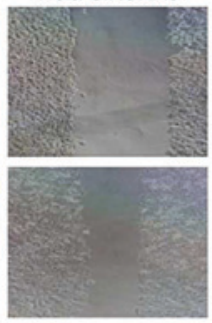

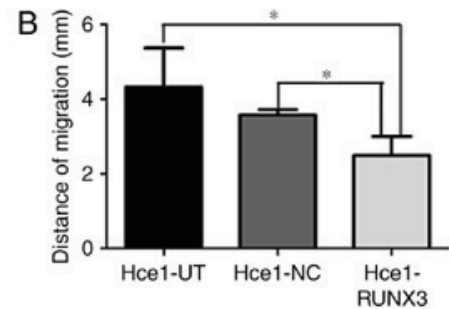

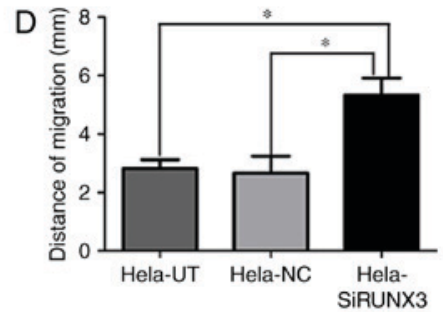

Figure 3. Migration of the cells to the wound was visualized at 0 and $48 \mathrm{~h}$. The distance between the edges of the scratches were measured after $48 \mathrm{~h}$. (A) The migration state of forced RUNX3 cell at 0 and $48 \mathrm{~h}$. Original magnification, x100. (B) Would healing assay showed the migration distance of forced RUNX3 cell after $48 \mathrm{~h}$ was much shorter than Hcel-UT and Hce1-NC groups. (C) The migration state of HeLa-SiRUNX3 cells at 0 and 48 h. Original magnification, x100. (D) The migration distance of HeLa-SiRUNX3 cells obviously longer than HeLa-UT and HeLa-NC group after 48 h. *P<0.05. UT, untreated; NC, negative control.

A

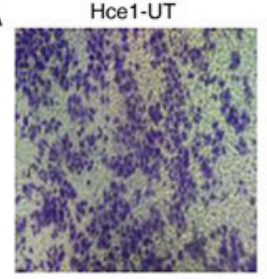

C

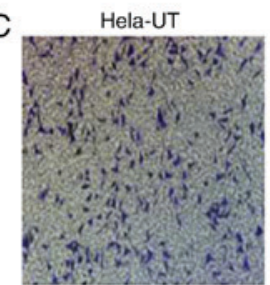

Hce1-NC
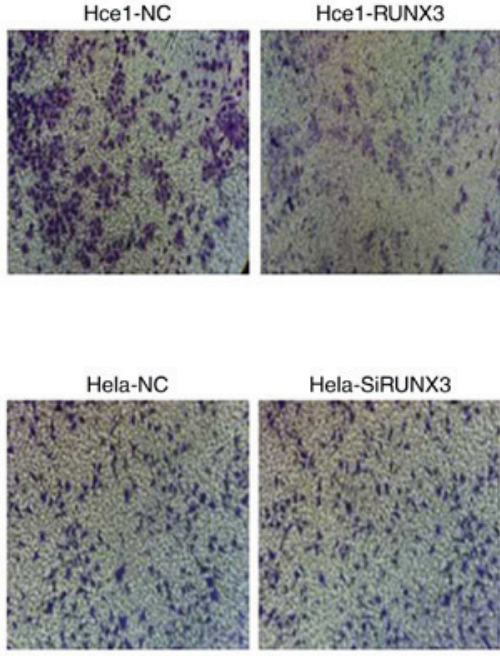

Hela-SiRUNX3

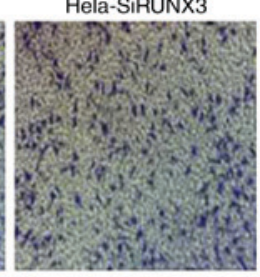

B
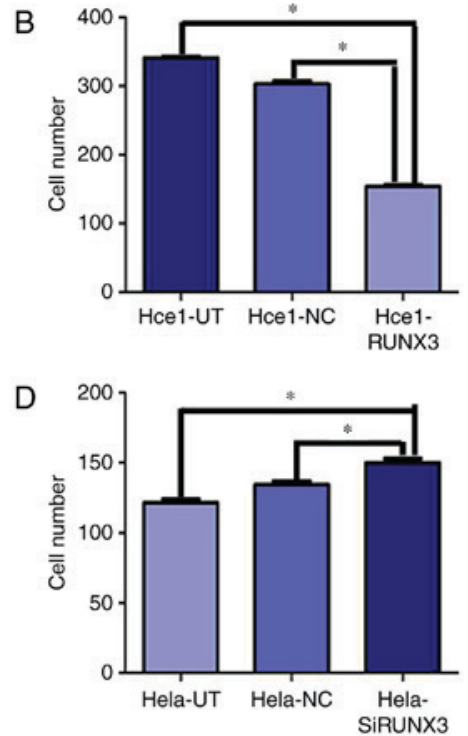

Figure 4. The effects of RUNX3 expression on cell invasion. (A and B) Hce1-RUNX3 cells showed less invasive ability than Hce1-UT and Hce1-NC groups. Original magnification, x200. (C and D) HeLa-SiRUNX3 cells performed stronger invasive ability than HeLa-UT and HeLa-NC groups. Original magnification, $\mathrm{x} 200 .{ }^{*} \mathrm{P}<0.05$. UT, untreated; NC, negative control.

\section{Discussion}

The HCE cell line is misidentified according to: http: //iclac. org/wp -content/uploads/Cross-Contaminations-v8_0.pdf, but the cell of HCE1 we used in our study names Hunan cervical epithelial cell line no. 1 (HCE1) was established from 5 carcinoma specimens of hysterectomy by Chinese researchers in 2000, which has been passaged more than 80 times, obviously different with HCE cell line mentioned in the data which reported in 1981 (20). Hence, the HCE1 cells in our study definitely not consistant with what the data mentioned. During the experiment, we strictly adhere to the rules of cell culture, the morphology and growth characteristics are quite difference between HCE1 and Hela cells. HCE1 cells have no possibility of being contaminated by Hela cells.

So far, only a few reports have addressed the biological behavior of RUNX3 in tumorigenesis of cervical cancer. The mechanisms by which RUNX3 acts in cervical cancer have not been reported. Transcription factor of RUNX3, which is a downstream effector of TGF- $\beta$, acts on the TGF- $\beta$ receptor type II (21) or the downstream protein SMADs, and then promotes the proliferation, apoptosis, angiogenesis, and invasion of tumor cells through TGF- $\beta$ signal transduction pathway (22-24), which might explain its wide involvement in tumorigenesis, including that of cervical cancer. 
A

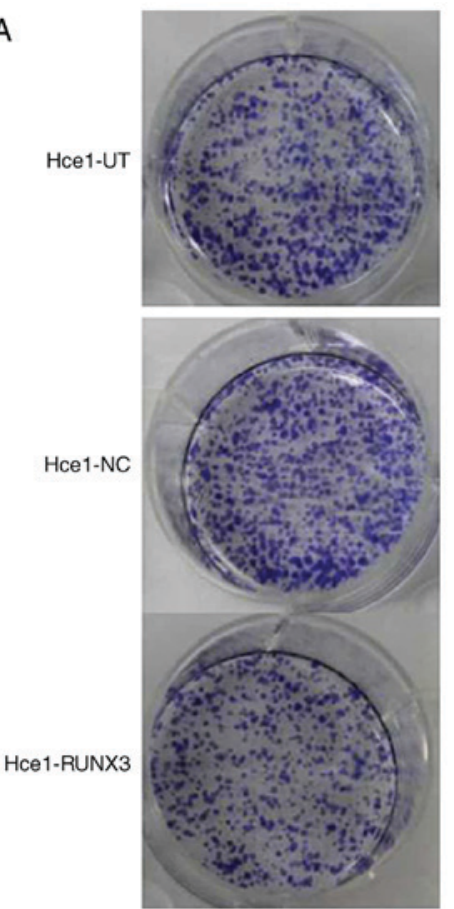

B

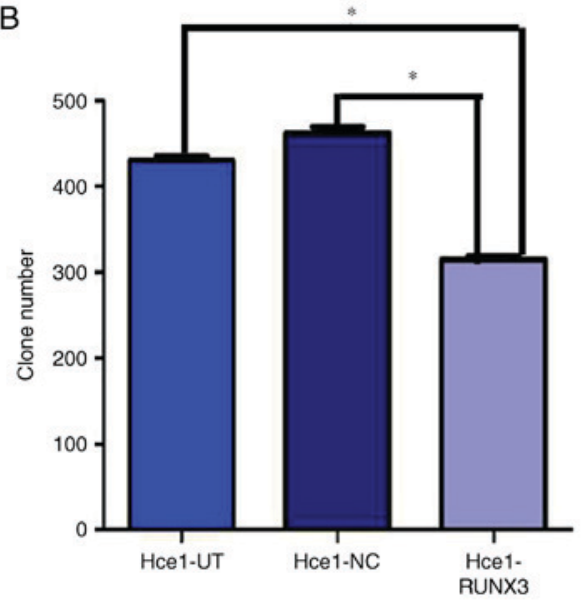

$\mathrm{C}$
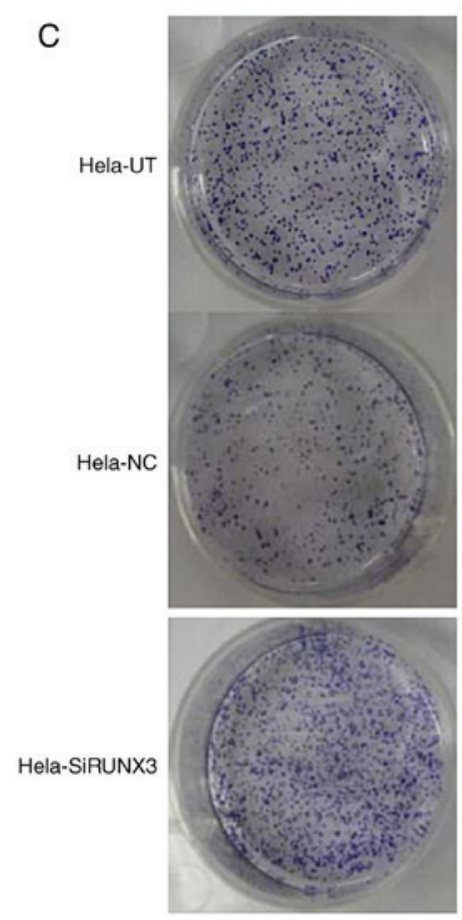

D

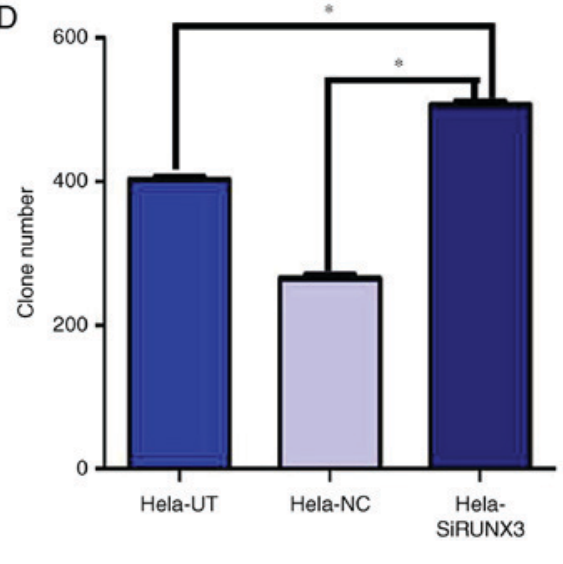

Figure 5. Role of RUNX3 expression in colony formation. (A and B) Representative results of colony formation assay produced by Hce1-RUNX3, Hce1-UT and Hcel-NC cells. (C and D) Representative results of colony formation assay produced by HeLa-SiRUNX3, HeLa-UT and HeLa-NC cells. *P<0.05. UT, Untreated; NC, Negative control.

In the present study, the recombination plasmid of PCDNA3.1- RUNX3 was constructed and successfully transfected into Hcel cells. The expressive efficiency of RUNX3 in Hce1-RUNX3 cells and Hela-SiRUNX3 cells were examined by western blot which showed significantly increases in Hce1-RUNX3 cells and markedly decreased in Hela-SiRUNX3 cells. Our results demonstrated that high levels of RUNX3 expression could effectively inhibit the migration and invasion of cervical cancer cells, compared with control group in Hcel cells. However, down-regulation of RUNX3 expression promoted migration and invasion by Hela cells. Moreover, colony formation numbers of cervical cancer cells decreased dramatically after transfection with RUNX3. In this way, our study showed that RUNX3 might play an important role in inhibiting the proliferation, migration, and invasion of cervical cancer cells.

Collectively, these results show that RUNX3 plays significant roles in the development of cervical cancer, and it might be a promising strategy for cervical cancer therapy. Further study of the underlying mechanisms and signaling pathways of RUNX3 in cervical cancer may help improve the prognosis of patients with cervical cancer.

\section{Acknowledgments}

Not applicable.

\section{Funding}

The present study was supported by grants from the National Natural Science Foundation of China (grant no. 81302245).

\section{Availability of data and materials}

All data generated or analyzed during this study are included in this published article. 


\section{Authors' contributions}

PF contributed to analysis and manuscript preparation. ZL and $\mathrm{CZ}$ performed the data analyses and wrote the manuscript. MD helped perform the analysis with constructive discussions.

\section{Ethics approval and consent to participate}

Not applicable.

\section{Consent for publication}

Not applicable.

\section{Competing interests}

The authors declare that they have no competing interests.

\section{References}

1. Lertkhachonsuk AA, Yip CH, Khuhaprema T, Chen DS, Plummer M, Jee SH, Toi M and Wilailak S; Asian Oncology Summit 2013: Cancer prevention in Asia: Resource-stratified guidelines from the Asian Oncology Summit 2013. Lancet Oncol 14: e497-e507, 2005.

2. Mohanty G and Ghosh SN: Risk factors for cancer of cervix, status of screening and methods for its detection. Arch Gynecol Obstet 291: 247-249, 2015.

3. Ito Y: RUNX genes in development and cancer: Regulation of viral gene expression and the discovery of RUNX family genes. Adv Cancer Res 99: 33-76, 2008.

4. Hug BA, Ahmed N, Robbins JA and Lazar MA: A chromatin immunoprecipitation screen reveals protein kinase Cbeta as a direct RUNX1 target gene. J Biol Chem 279: 825-830, 2004.

5. Ito $\mathrm{Y}$ and Miyazono K: RUNX transcription factors as key targets of TGF-beta superfamily signaling. Curr Opin Genet Dev 13: 43-47, 2003.

6. Bangsow C, Rubins N, Glusman G, Bernstein Y, Negreanu V, Goldenberg D, Lotem J, Ben-Asher E, Lancet D, Levanon D and Groner Y: The RUNX3 gene-sequence, structure and regulated expression. Gene 279: 221-232, 2001.

7. Wang Y, Feng Q, Ji C, Liu X, Li L and Luo J: RUNX3 plays an important role in mediating the BMP9-induced osteogenic differentiation of mesenchymal stem cells. Int J Mol Med 40: 1991-1999, 2017.

8. Li QL, Ito K, Sakakura C, Fukamachi H, Inoue Ki, Chi XZ, Lee KY, Nomura S, Lee CW, Han SB, et al: Causal relationship between the loss of RUNX3 expression and gastric cancer. Cell 109: 113-124, 2002.
9. Chen LF: Tumor suppressor function of RUNX3 in breast cancer. J Cell Biochem 113: 1470-1477, 2012.

10. Vogiatzi P, De Falco G, Claudio PP and Giordano A: How does the human RUNX3 gene induce apoptosis in gastric cancer? Latest data, reflections and reactions. Cancer Biol Ther 5: 371-374, 2006.

11. Lee SH, Kim J, Kim WH and Lee YM: Hypoxic silencing of tumor suppressor RUNX3 by histone modification in gastric cancer cells. Oncogene 15: 184-194, 2009.

12. Suzuki M, Shigematsu H, Shames DS, Sunaga N, Takahashi T, Shivapurkar N, Iizasa T, Frenkel EP, Minna JD, Fujisawa T and Gazdar AF: DNA methylation-associated inactivation of TGFbeta-related genes DRM/Gremlin, RUNX3 and HPP1 in human cancers. Br J Cancer 93: 1029-1037, 2005.

13. Nevadunsky NS, Barbieri JS, Kwong J, Merritt MA, Welch WR, Berkowitz RS and Mok SC: RUNX3 protein is overexpressed in human epithelial ovarian cancer. Gynecol Oncol 112: 325-330, 2009.

14. Lee CW, Chuang LS, Kimura S, Lai SK, Ong CW, Yan B, Salto-Tellez $M$, Choolani $M$ and Ito $Y$ : RUNX3 functions as an oncogene in ovarian cancer. Gynecol Oncol 122: 410-417, 2011.

15. Tsunematsu T, Kudo Y, Iizuka S, Ogawa I, Fujita T, Kurihara H, Abiko Y and Takata T: RUNX3 has an oncogenic role in head and neck cancer. PLoS One 4: e5892, 2009.

16. Kudo Y, Tsunematsu T and Takata T: Oncogenic role of RUNX3 in head and neck cancer. J Cell Biochem 112: 387-393, 2011.

17. Lai KW, Koh KX, Loh M, Tada K, Subramaniam MM, Lim XY, Vaithilingam A, Salto-Tellez M, Iacopetta B and Ito Y: MicroRNA-130b regulates the tumour suppressor RUNX3 in gastric cancer. Eur J Cancer 46: 1456-1463, 2010.

18. Zheng F, Wu J, Zhao S, Luo Q, Tang Q, Yang L, Li L, Wu W and Hann SS: Baicalein increases the expression and reciprocal interplay of RUNX3 and FOXO3a through crosstalk of AMPKo and MEK/ERK1/2 signaling pathways in human non-small cell lung cancer cells. J Exp Clin Cancer Res 34: 41, 2015.

19. Lee YS, Lee JW, Jang JW, Chi XZ, Kim JH, Li YH, Kim MK, Kim DM, Choi BS, Kim EG, et al: RUNX3 inactivation is a crucial early event in the development of lung adenocarcinoma. Cancer Cell 24: 603-616, 2013.

20. Wu SF, Zhu HC, Gu FH, Huang BY and Liu HY: Characteristics of an established cervical carcinoma cell line HCE1. Hunan Yi Ke Da Xue Xue Bao 25: 532-534, 2000 (In Chinese).

21. Ito K, Lim AC, Salto-Tellez M, Motoda L, Osato M, Chuang LS, Lee CW, Voon DC, Koo JK, Wang H, et al: RUNX3 attenuates beta-catenin/T cell factors in intestinal tumorigenesis. Cancer Cell 14: 226-237, 2008.

22. Tang B, Du J, Li Y, Tang F, Wang Z and He S: EZH2 elevates the proliferation of human cholangiocarcinoma cells through the downregulation of RUNX3. Med Oncol 31: 271, 2014.

23. Subramaniam MM, Chan JY, Yeoh KG, Quek T, Ito K and Salto-Tellez M: Molecular pathology of RUNX3 in human carcinogenesis. Biochim Biophys Acta 1796: 315-331, 2009.

24. Chen F, Liu X, Bai J, Pei D and Zheng J: The emerging role of RUNX3 in cancer metastasis (Review). Oncol Rep 35: 1227-1236, 2016. 